\title{
LAMOST Medium-Resolution Spectral Survey of Galactic Nebula (LAMOST MRS-N): An overview of Scientific goals and Survey plan
}

Chao-Jian $\mathrm{Wu}^{1,3}$, Hong $\mathrm{Wu}^{1,3}$, Wei Zhang ${ }^{1,3}$, Juan-Juan Ren ${ }^{2,3}$, Jian-Jun Chen ${ }^{1,3}$, Chih-Hao $\mathrm{Hsia}^{4}$, Yu-Zhong Wu ${ }^{1,3}$, Hui Zhu ${ }^{3}$, Bin Li ${ }^{5,6}$, Yong-Hui Hou ${ }^{7,8}$, Jun-Lin Wang ${ }^{4}$, Shuo-Ran $\mathrm{Yu}^{4}$, LAMOST MRS Collaboration

1 CAS Key Laboratory of Optical Astronomy, National Astronomical Observatories, Chinese Academy of Sciences, Beijing 100101, China; chjwu@bao.ac.cn

2 CAS Key Laboratory of Space Astronomy and Technology, National Astronomical Observatories, Chinese Academy of Sciences, Beijing 100101, China

3 National Astronomical Observatories, Chinese Academy of Sciences, 20A Datun Road, Chaoyang District, Beijing 100101, China

4 State Key Laboratory of Lunar and Planetary Sciences, Macau University of Science and Technology, Taipa, Macau, China

5 Purple Mountain Observatory, Chinese Academy of Sciences, Nanjing 210008, People's Republic of China

6 University of Science and Technology of China, Hefei 230026, People's Republic of China

7 Nanjing Institute of Astronomical Optics, \& Technology, National Astronomical Observatories, Chinese Academy of Sciences, Nanjing 210042, China

8 School of Astronomy and Space Science, University of Chinese Academy of Sciences

Received —; accepted -

\begin{abstract}
Since Sep. 2018, LAMOST started the medium-resolution $(\mathrm{R} \sim 7500)$ spectral survey (MRS). We proposed the spectral survey of Galactic nebulae, including H II regions, $\mathrm{HH}$ objects, supernova remnants, planetary nebulae and the special stars with MRS (LAMOST MRS-N). LAMOST MRS-N covers about 1700 square degrees of the northern Galactic plane within $40^{\circ}<l<215^{\circ}$ and $-5^{\circ}<b<5^{\circ}$. In this 5-year survey, we plan to observe about 500 thousand nebulae spectra. According to the commissioning observations, the nebulae spectra can provide precise radial velocity with uncertainty less than $1 \mathrm{~km} \mathrm{~s}^{-1}$. These high-precision spectral data are of great significance to our understanding of star formation and evolution.
\end{abstract}

Key words: surveys — ISM: general — ISM: HII regions — ISM: HerbigHaro objects — ISM: planetary nebulae: — ISM: supernova remnants — Galaxy: disk

\section{INTRODUCTION}

The Milky Way is a unique laboratory for studying the formation of stars and the structure of the universe. According to the Hubble classification, the Milky Way is a typical barred spiral galaxy, which consists of disk, nuclear sphere and halo (Gerhard, 2002). The Galactic plane (GP) contains majority of baryonic matter in the Milky Way, and it is the main region of star formation and evolution. A large 
number of stars are born in the nebulae of the GP. The study of nebulae is of great significance to understand the formation and evolution of stars and the feedback of interstellar matter. Through the spectral observation of the nebulae on the GP, we can obtain the basic physical parameters, including distance, mass, velocity, electronic temperature and density, abundance of many chemical elements, and other physical parameters of the ionized gas around the star, we can also study the physical properties of the accretion disk, accretion process of the protoplanetary disk and dynamic information. Moreover, the physical mechanism and evolution process of interstellar medium (ISM) in the early and late stages of star formation can also be studied. However, there is not yet a complete spectral survey of emission nebulae on the GP. Large Sky Area Multi-Object fiber Spectroscopic Telescope (LAMOST), similar to Integral Field Unit (IFU) type structure, provides us an opportunity to make spectral observations for a large number of Galactic nebulae.

The LAMOST, also known as Guoshoujing Telescope, is a 4-meter effective aperture quasimeridian reflecting Schmidt telescope, which is equipped with 4000 fibers on its $5^{\circ}$ diameter field of view focal plane. It is the first large astronomical scientific device in China (Cui et al., 2012; Zhao et al., 2012; Wang et al., 1996; Su \& Cui, 2004). Until 2017, LAMOST has completed its pilot and first 5 years low spectral resolution $(\mathrm{R} \sim 1800)$ regular sky survey and achieved great success. During 2017, another new 16 medium spectral resolution $(\mathrm{R} \sim 7500)$ spectrographs were installed on LAMOST (Liu et al., 2020). The two cameras (blue and red) of each spectrograph can cover the wavelength range $4950 \AA$ $5350 \AA$ and $6300 \AA-6800 \AA$, respectively (Wu et al., 2020; Liu et al., 2020). The red part of the spectra can well cover $\mathrm{H} \alpha$, [N II] $\lambda \lambda 6548,6584$ and [S II] $\lambda \lambda 6717,6731$ emission lines, while the blue part can well cover [O III] $\lambda \lambda 4959,5007$ emission line. The six emission lines are all crucial for the Galactic nebulae.

Since Sep. 2018, LAMOST started the second stage survey (the Medium-Resolution Spectral Survey, MRS) program. We proposed the spectral survey of Galactic nebulae with LAMOST MRS (hereafter: MRS-N). In addition to MRS-N, another several scientific goals of MRS are Galactic archaeology, stellar physics, star clusters, star formation, exoplanet and their host stars etc.

The structures of this paper are as follow: Section 2 describes the proposed scientific goals. Section 3 provides a detailed report of the survey strategy, input catalog, progress and preliminary results. And Section 4 gives a brief summary.

\section{SCIENCE}

The main scientific goals of our MRS-N project focus on four-type nebulae including H II regions, Herbig-Haro objects, supernovae remnants and planetary nebulae. We aim to obtain the three-dimension (3D) properties of these nebulae such as radial velocities, velocity dispersions, reddening and chemical compositions, which can help us to study the environments surrounding star formation regions and evolved stars and to understand the feedback of star formation on the dynamics and energetics of Galactic interstellar medium (ISM). Compared to the low-resolution spectra, the medium resolution spectra can provide more accurate velocity measurements, and are more efficient to separate different components along the same line-of-sight of the nebulae based on their various velocity distributions.

Besides nebulae, some individual objects such as Be stars (Wu et al., 2020), F and OB stars, luminous blue variables (LBVs), red supergiants (RSGs), and Wolf-Rayet stars are also the scientific goals of our MRS-N project.

\subsection{H II regions}

H II regions are the diffuse nebulae with amorphous photoionized by ultraviolet radiations emitted from young massive stars. As the radiation sources are short-lived stars, and most of the gas are swept away by strong radiation pressure, the $\mathrm{H}$ II regions will fade away after a few million years Kennicutt \& Chu, 1988). As the distribution of these stars and the surrounding gas are irregular, in most situation, the shape of the H II regions is far from simple Stromgren sphere, instead, they often appear clumpy and filamentary (Monreal-Ibero et al., 2011). From the optical spectra, we can easily identify strong hydrogen emis- 
sion lines (such as $\mathrm{H} \alpha, \mathrm{H} \beta$ ) and fine-structure lines (such as [O II] $\lambda \lambda 3726,3729$, [O III] $\lambda \lambda 4959,5007$, [N II] $\lambda \lambda 6548,6584$, [S II] $\lambda \lambda 6717,6731)$, which are usually used to derive current star formation rate, internal reddening, metallicities, the electron temperature and densities of the nebulae (Pérez-Montero, 2017). So the H II regions are natural laboratories for studying star-forming actives and interactions between the massive stars and surrounding ISM (Mao et al., 2018).

The spatial variation of chemical abundances across the galactic disc is an observational constraint for chemical evolution models. Galactic abundance gradients in the ISM were described by Searle (1971) in a survey of HII regions in six Sc galaxies. Shaver et al. (1983) firstly showed that the abundances were not distributed homogenously throughout the disc of the Milky Way. Since then radial abundance gradients probed by H II regions have been studied extensively (Simpson et al., 1995; Afflerbach et al., 1997; Deharveng et al., 2000; Esteban et al., 2005; Rudolph et al., 2006; Balser et al., 2011; Fernández-Martín et al., 2017; Esteban et al., 2017), and the results showed that the abundances were decreasing with Galactocentric distances, indicating that the inside-out models of galaxy formation are valid to explain the chemical composition of the Milky Way (Chiappini et al., 1997, 2001).

Whether the slope is uniform along the entire disc is still an open question. Some studies found there is a flatter radial abundance gradient at the outer disc (Fich \& Silkev, 1991; Vilchez \& Esteban, 1996; Esteban et al., 2013; Korotin et al., 2014; Fernández-Martín et al., 2017), while some others did not (Deharveng et al., 2000; Rudolph et al., 2006; Esteban et al., 2017). Most recently, using H II regions in the Galactic anti-center area from LAMOST DR5 and several H II regions from literatures, Wang et al. (2018) determined the slopes of oxygen abundance gradient of inner and outer parts, and claimed the absence of flattening at the outer disc.

The Galactic plane survey of LAMOST-MRS-N is in the coverage of the Isaac Newton Telescope Photometric H-Alpha Survey of the Northern Galactic Plane (IPHAS) (Drew et al., 2005; Barentsen et al., 2014). By assigning the fibers to diffuse regions of bright $\mathrm{H} \alpha$ emission, the LAMOSTMRS-N will obtain large numbers of optical spectra of H II regions on the Galactic disc, which allow us to study the radial abundance gradient and to re-check whether there is evidence of flattening at the outer disc. By comparing the results with chemical evolution models, our understanding of formation and evolution of the Milky Way will be enchanced.

One important intrinsic characteristic of giant H II regions (diameter $>100 \mathrm{pc}$ and $\mathrm{H} \alpha$ luminosity $\left.\sim 10^{39} \mathrm{erg} \mathrm{s}^{-1}\right)$ is that they present supersonic velocity dispersions $\left(\sigma \geq 12.85 \mathrm{~km} \mathrm{~s}^{-1}\right)$ (Smith \& Weedman, 1970; Fuentes-Masip et al., 2000). There are mainly two kinds of possible mechanisms to explain the supersonic line widths, one is gravitational models, in which the Gaussian line profiles are assumed to be related to virialized motions within the H II regions (Terlevich \& Melnick, 1981), another one is wind-driven models, in which the emission line profiles are broadened by mechanical energy injected by massive stars (Dyson, 1979; Tenorio-Tagle et al., 1996).

\subsection{Herbig-Haro objects}

Herbig-Haro objects (HH objects, (Ambartsumian, 1954) ) are formed when narrow jets of partially ionized gas ejected by stars collide with nearby gas and dust at speeds of several hundred kilometres per second. There are more 1000 individual objects have been discovered(Davis et al., 2010). They are small-scale shock regions intimately associated with star forming regions (Schwartz, 1983) and can trace supersonic shock waves (Bally, 2016), often found in large groups, and some can be seen around a single star, aligned with its rotational axis.

$\mathrm{HH}$ objects show strong hydrogen recombination lines and various forbidden lines, in particular [S II] and [O II], but no detectable optical continuum emission. Their nature and origin remained a puzzle over quite some time, although it was clear from the beginning that they were somehow connected to star formation. A major step towards an understanding of $\mathrm{HH}$ objects came with the suggestion that their spectral properties might arise in gas that is shock excited by supersonic winds from the young stars (Schwartz, 1975). The next crucial step was the discovery of the high proper motions in HH objects indicative of space motions of several hundred $\mathrm{km} \mathrm{s}^{-1}$ (Cudworth \& Herbig, 1979). Another observation led to the still widely accepted basic picture of what the majority of HH objects are: most 
$\mathrm{HH}$ objects were not independent entities (like bullets), but shock fronts in continuous, well collimated jets driven by young stellar objects (Mundt, 1985, 1987).

The common methods for studying $\mathrm{HH}$ objects are narrow band photometry and spectral observation. While the narrow band images can provide the information of only one specific band each time. To study HH objects in detail, many narrow band filters are needed, at the same time, more observation time will be taken. While the spectral observations are more efficient. Fortunately, LAMOST-MRS-N provides the opportunity to study the most of known $\mathrm{HH}$ objects in detail and identify more $\mathrm{HH}$ objects candidates.

\subsection{Supernovae Remnants}

Supernova Remnants (SNRs) are the material ejected in an explosion of supernovae, which can continue their life through the interactions with the surrounding interstellar medium (ISM) for thousands or even up to a million years. SNRs are also the important sources of the injection of energy and heavy elements into the ISM, and are believed to be the possible sources of the Galactic cosmic rays. Moreover, SNRs probe the immediate surroundings of supernovae, shaped by their progenitors. SNRs are generally characterized by the interaction of supernova ejecta with the surrounding ISM. The main features of SNRs are strong shock waves, amplified magnetic field, ultra-relativistic particles (cosmic rays) generation and associated synchrotrion radiation (Arbutina, 2017). Currently, 294 Galactic SNRs (Green, 2014) have been discovered. A great majority $(\sim 79 \%)$ of the known Galactic SNRs are believed to be relatively old with ages greater than $10^{3}$ years and radii larger than $\sim 5 \mathrm{pc}$, and are evolved objects in either the adiabatic or the early radiative stages of their evolutionary development (Woltier, 1972). These old and evolved SNRs generally show characteristic shell structures (Fesen et al., 1985). About 30\% of Galactic SNRs are known to have optical emission associated with their nonthermal radio emission. For old remnants, the optical emission arises from the cooling of shocked interstellar cloud material following the passage of the remnant's blast waves as they expand outward into the ambient medium.

Optical spectra of SNRs usually show strong emission including that of $\mathrm{H} \alpha$, [O II] $] \lambda \lambda 3726,3729$, [O III] $\lambda \lambda$ 4959,5007, [N II] $\lambda \lambda$ 6548,6584 and [S II] $\lambda \lambda$ 6717,6731. And the line intensity ratios have important physical implications. The optical spectra of SNRs have elevated [S II]/H $\alpha$ emission-line ratios, as compared to the spectra of normal H II regions, and through this technique, more than 1000 optical extra-Galactic SNR candidates have been detected so far (Vučetić et al., 2015). The [S II]/H $\alpha$ ratio can be also used to differentiate the shock-heated SNRs (ratios $>0.4$, but often considerably higher) and photoionized nebulae $(0.4$, but typically $<0.2)$. As in typical $\mathrm{HII}$ regions, sulfur exists mainly in the form of S III, thus the $[\mathrm{SII}] / \mathrm{H} \alpha$ ratio is very low. While after the shock wave from an SN explosion has propagated through the surrounding medium, and the material has cooled sufficiently, a variety of ionization states can be present, including the [S II], thus leading to the increased ratio $[\mathrm{S} I I] / \mathrm{H} \alpha$ observed in SNRs (Blair \& Long, 2004). Generally, in H II regions the material is too ionized to produce forbidden lines of [O I], [N II] or [S II], while in SNRs, both the models of Allen et al. (2008) and observations show optical spectra containing these forbidden lines (Long (2016)). Furthermore, the [S II]6717/[S II]6731 ratio is electron density sensitive, thus can be used to obtain the electron density. The $\mathrm{H} \alpha /[\mathrm{N} \mathrm{II}] 6584$ ratio is a widely used tool for investigating nitrogen-to-hydrogen abundance variations among SNRs. Thus it is important to obtain complete sampling of the optical spectra covering these emission in SNRs.

Although several large and evolved SNRs have been well studied morphologically in the optical, only a limited amount of spectroscopic data are available for those faint optical remnants, especially for those with large angular sizes. While many SNRs are very large, where there are 10 large SNRs with angular sizes greater than $2^{\circ}$ (Orchiston et al., 2015). It would be extremely time consuming to obtain spectra covering such a large spatial extent using regular telescopes. The wide field of view, multi-fiber survey telescope LAMOST thus serves as a perfect tool for mapping the details of those extremely extended SNRs. Ren et al. (2018) has tried to map a large SNR (the S147 with angular diameter $\sim 200$ arcmin) with the LAMOST low-resolution $(\mathrm{R} \sim 1800)$ spectra, but due to the low-resolution, the details can not be mapped clearly. With this increased higher resolution of MRS-N, more detailed emission 
features and kinematics properties can be mapped for SNRs, thus leading to better understandings of the properties of SNRs.

\subsection{Planetary nebulae}

Planetary nebulae (PNs) are originally thought to be a member of $\mathrm{H}$ II regions, in fact they are completely different from normal emission nebulae. PNs are the evolutionary end products of low- and intermediatemass stars $\left(1 \sim 8 \mathrm{M}_{\odot}\right)$. The total number of Galactic PNs is about 2860 , including $\sim 1500$ in the Galactic PN Catalogue of Kohoutek (2001), 1200 PNs listed in the Macquarie-AAO-Strasbourg H $\alpha$ Catalogue of Galactic PNs (MASH; Parker et al. (2006); Miszalski et al. (2008)), and 160 faint extended PNs discovered by the IPHAS. Most PNs are located close to the Galactic plane and suffer larger interstellar extinction. They always show low surface brightness and can be easily mixed with background diffuse nebulae or star formation regions.

With the great help of MRS-N, a significant fraction of known PNs ( $\sim 14 \%$; 400 PNs) will be observed for studying their 3D ionized structures based on various nebular expansion velocities. The gaseous structures of Galactic PNs and surrounding nebulae are easily separated by their various spatial expansion velocities. In addition, some foundational properties of these PNs can also be derived. The survey provides us an opportunity to study 3D ionized structures and electron density distributions of Galactic PNs, the evolution of various morphological PNs, and the chemical abundance of our galaxy.

MRS with large spatial coverage and high sensitivity as a powerful tool to study PNs obscured in the GP. Many new PNs expected to discover through this survey, containing young, compact PNs and old, extended, faint PNs. The nature and the PN status of these nebulae can be identified using the $\log (\mathrm{H} \alpha /[\mathrm{N} \mathrm{II}])$ vs. $\log (\mathrm{H} \alpha /[\mathrm{S} \mathrm{II}])$ emission-line-ratio diagnostic diagram (Hsia \& Zhang, 2014), which is an useful probe to distinguish PNs from H II regions and supernova remnants (SNRs) with ionized gaseous nebulae.

\section{SURVEY PLAN}

\subsection{Strategy}

Most nebulae are located on the GP. So the survey areas of MRS-N mainly cover the northern GP. The northern GP is within the Galactic longitude range $40^{\circ}<l<215^{\circ}$ and the latitude range $-5^{\circ}<b<5^{\circ}$, totally about 1700 square degrees. Furthermore, four large size nebulae are selected to be observed with MRS as the specific areas in our Galaxy. The four specific areas are Westerhout 5 (H II region, hereafter West), Rosettle Nebula+NGC2264 (H II region, hereafter Ros), Cygnus Loop (SNRs, hereafter Cyg) and Simeis 147 (SNRs, hereafter S147), respectively.

In MRS-N, we plan to use 123 plates cover the GP areas (blue circles in Figure 11. 11 shows that the MRS-N coverage can cover most of $\mathrm{H}$ II region and SNRs in the northern celestial sphere. A plate, covering about $20 \mathrm{deg}^{2}$ (a circle with a diameter of $5^{\circ}$ ), will continuously take three single $1200 \mathrm{~s}$ (900 $\mathrm{s}$ for MRS-N, the exposure time of $900 \mathrm{~s}$ can provide enough high signal-to-noise ratio nebulae spectra) exposure and obtain the co-added spectra with total exposure time of $3 \times 1200 \mathrm{~s}(3 \times 900 \mathrm{~s}$ for MRS-N). For each specific area, the exposure time is designed as $16 \times 900 \mathrm{~s}$ (red circles in Figure 1). So it will take about 130 hours to finish the whole MRS-N survey. MRS-N will be assigned 30 hours per year. So we could finish the MRS-N survey within 5 years without considering other objective factor such as weather.

The commissioning observations were carried out on Mar. 4-5, 2018. Figure 2 (a) shows the fiber distribution. More detailed observation information are listed in Table 1 The results showed that MRS$\mathrm{N}$ is seriously affected by the moonlight. The spectrum of one fiber (red plus in Figure $2 b$ ) is presented in Figure 3 The $\mathrm{H} \alpha$ absorption and emission line at $6563 \AA$ are obviously mixed together. However, the absorption lines are not visible at $[\mathrm{N} \mathrm{II}](\lambda \lambda$ 6548,6584) and $[\mathrm{S} \mathrm{II}](\lambda \lambda 6717,6731)$. In fact, the $\mathrm{H} \alpha$ absorption line comes from the moonlight, to be exact, from the sunlight. On closer examination, we found that the spectrum was no longer affected when the altitude of the moon was lower than $1^{\circ}$. So all 


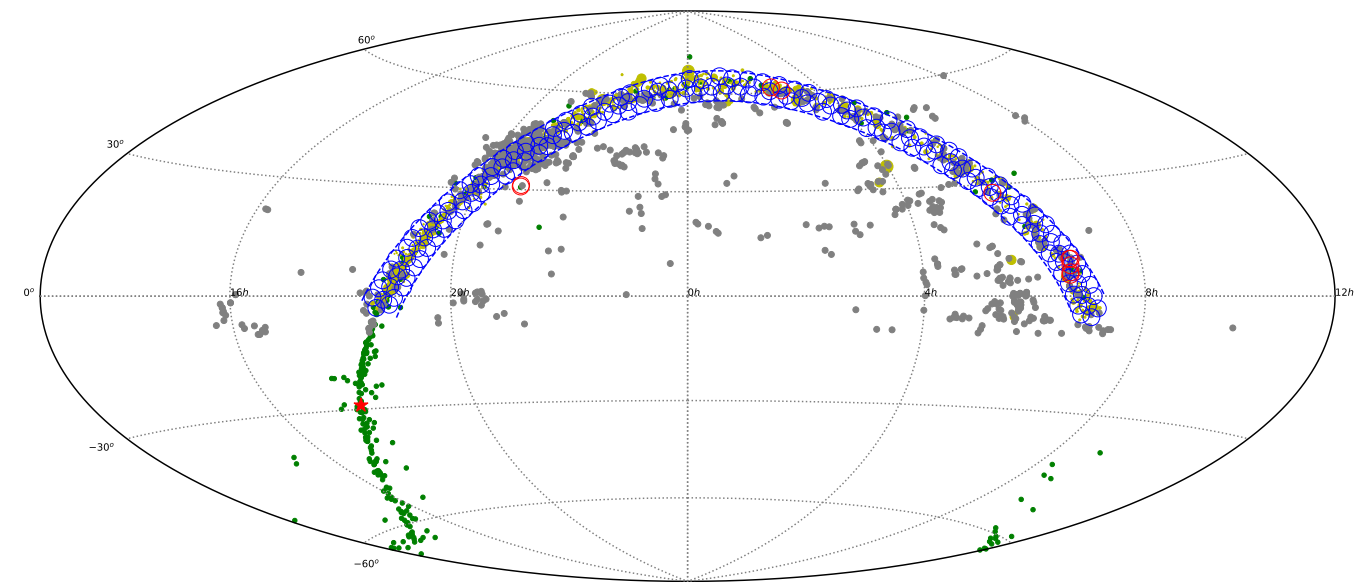

Fig. 1123 GP plates (blue circles) and four specific plates (red circles) for MRS-N in an Equatorial Aitoff projection. Most of the bright nebulae in the Milk way are marked with gray dots. The green dots represent the SNRs and the gray ones represent the H II region. The red star is the location of the Galactic center.

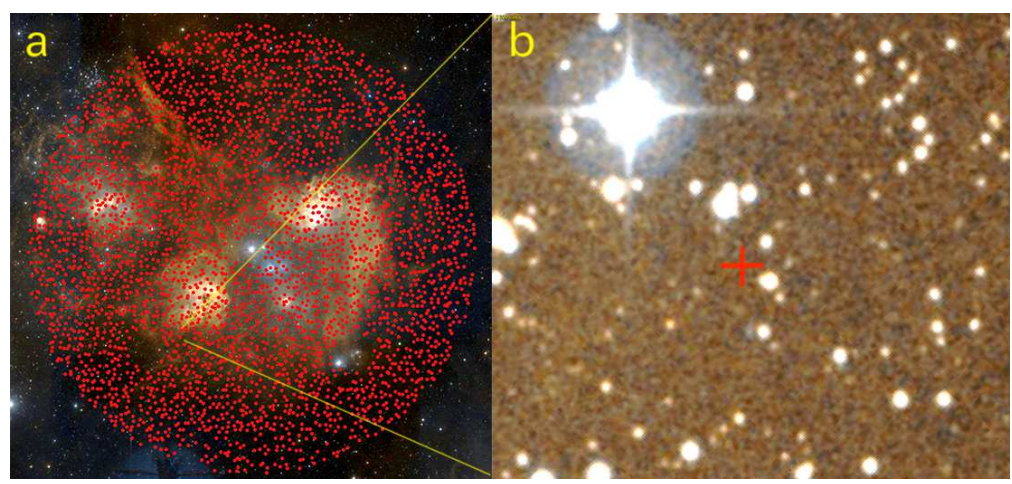

Fig. 2 (a): Fiber distribution of the commissioning observation on Mar. 4, 2018. (b): One fiber pointing to the nebula, its spectrum is drawn in Figure 3

the observation time of MRS-N should be assigned into a time range before the moon rises or after the moon sets. This will cause the observation time of MRS-N to be very urgent, which is why we adjust the sub-exposure time to $900 \mathrm{~s}$.

\subsection{Input catalog}

The input catalog is very important to LAMOST, which can be used to allocate the fiber's coordinates. MRS-N mainly focus on the spectra of non-point source (such as the structure of nebulae). There is not yet a catalog containing the structure of nebulae. Therefore, the input catalogs of MRS-N should be made independently unlike other MRS projects. 


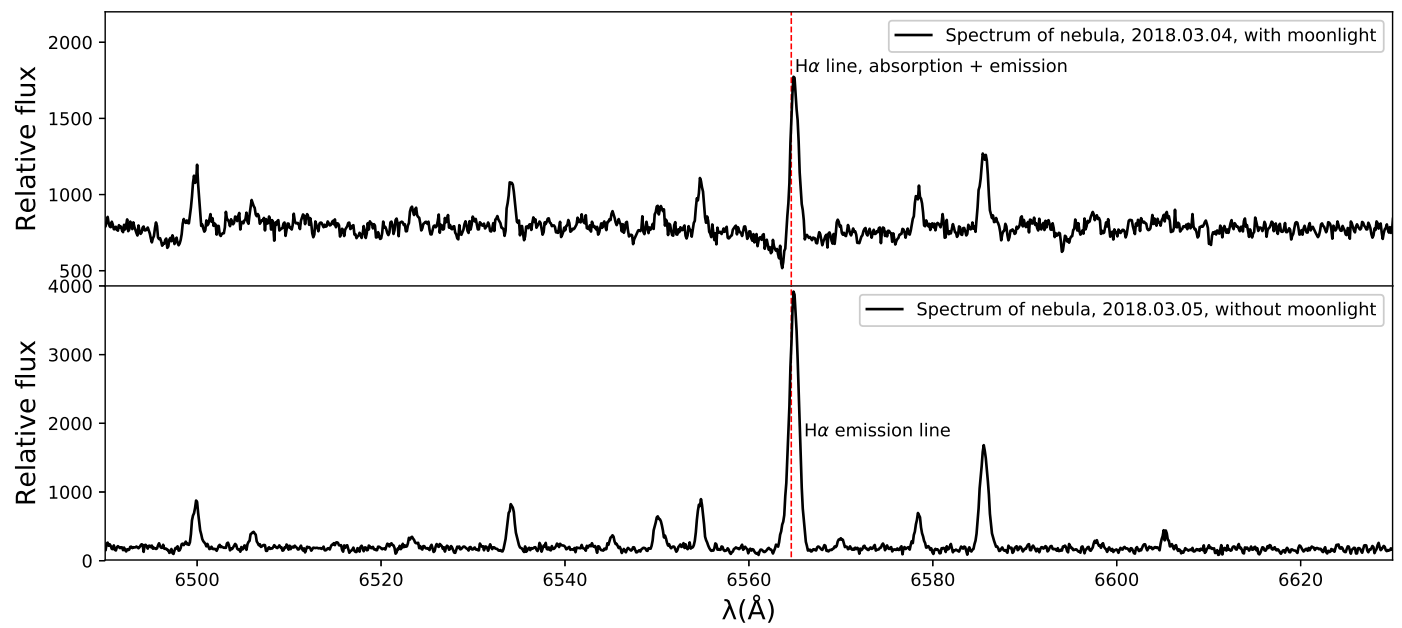

Fig. 3 Two spectra of commissioning observation. The upper panel shows a nebula spectrum observed on Mar. 4, 2018, when the moon had already risen. It is clearly to see that the $\mathrm{H} \alpha$ absorption and emission lines at $6563 \AA$ were mixed together. The bottom panel shows the nebula spectrum observed on Mar. 5, 2018, before the moon rose.

Table 1 The commissioning observation of MRS-N

\begin{tabular}{cccc}
\hline Date & Time $(\mathrm{UT})$ & Exposure $(\mathrm{s})$ & Alt of Moon \\
\hline 2018-03-04 & $13: 19: 32$ & 900 & $+6.5^{\circ}$ \\
2018-03-05 & $13: 01: 53$ & 900 & $-8^{\circ}$ \\
\hline
\end{tabular}

The input catalogs of four specific areas are man-made by comparing with optical images from the Digitized Sky Surey (DSS) and Narrow Band Survey (NBS). From optical images, some bright stars, some sky areas and the positions of interesting structure of nebulae are selected to compose input catalogs.

The input catalog of GP area is very complex. We divide the northern GP area into about 1.5 million $2^{\prime} \times 2^{\prime}$ grids. Each grid can be considered as the moving area of each fiber. Then we cross-match the 1.5 million grids with Hipparcos main catalog and Pan-STARRS1 (PS1). If a star within a grid is brighter than $13 \mathrm{mag}$ ( $\mathrm{r}$ band), then the star is selected as location of fiber with objtype STAR. By comparing with IPHAS and NBS images, if a star within a grid is bright than $18 \mathrm{mag}$ and fainter than $13 \mathrm{mag}$, then the location of fiber within the same grid should avoid the star with objtype skylight or $N E B$ (Nebulae). Else if there is not a star in a grid or the star is too faint to be observed, then the location of fiber should be pointed to the brightest position with objtype $N E B$ within the grid (Figure 4). In addition, we integrated some F stars, OB stars and emission stars from IPHAS into the input catalog of GP area.

Eventually, we finished a complete input catalog of MRS-N with more than 1.5 million coordinates.

\subsection{Data description and Preliminary results}

The data processing of MRS-N is different from LAMOST 1D pipeline (Luo et al., 2012, 2015). LAMOST 1D pipeline gives the physical parameters after deducting the skylight background. However, the method of deducting skylight background is not applicable to the nebulae spectra. How to reduce 


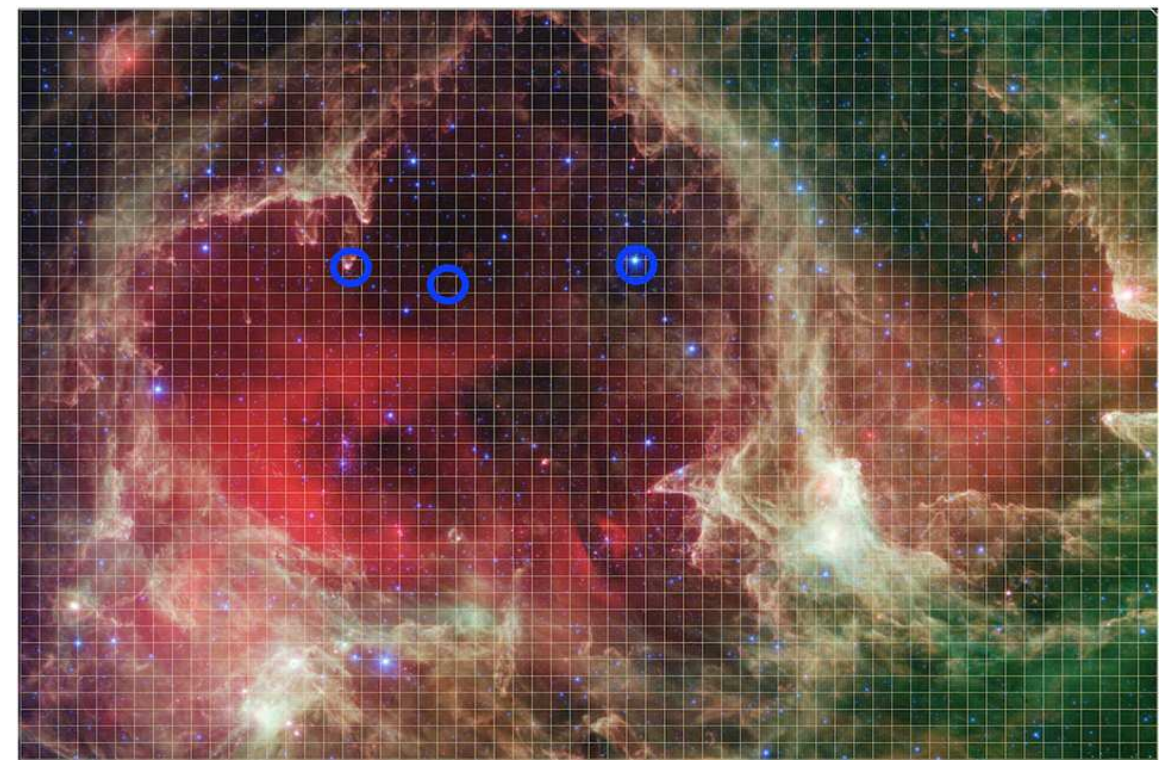

Fig. 4 Creating the input catalog of MRS-N. The size of each grid is $2^{\prime} \times 2^{\prime}$. If a star within a grid is brighter than 13 magnitude ( $\mathrm{r}$ band), then the star is selected as location of fiber (right blue circle), else if there is not a star in a grid or the star is too faint to observe, the location of fiber will be pointed to the brightest position (left and middle blue circle). The background image is Westerhout 5 .

the skylight in the spectra of the nebula is still under study. Therefore, MRS-N needs to have its own pipeline. The original nebulae spectra we get are the 1D spectra given by LAMOST 2D pipeline. The process includes dark and bias subtraction, flat field correction, spectral extraction, the first wavelength calibration. The MRS-N pipeline developed by MRS-N team includes deduction of cosmic rays, merging sub-exposures, fitting skylight emission line, reducing skylight background (optional), secondary wavelength calibration and parameters measurement of nebula emission line (such as radial velocity, intensity ratio of emission line, relative flux, line width, intensity and so on). More details about pipeline, data processing and data release will be presented in Wu et al. (2020 in prep).

Due to the more complex data processing, the data release of MRS-N is one year later than that of LAMOST. According to the LAMOST data release policy, the official data release has a protection period of 18 months. That is to say, the nebulae spectra of first year (from the second half of 2018 to the first half of 2019) will be officially released in March 2021, the rest will be done in the same manner. It is important to note that the nebula spectra in LAMOST DR7 has been processed by subtracting the skylight background and the results are not up to the release standard of MRS-N. If someone want to use nebula spectra for science, they should use the data released by MRS-N.

Figure 5, 6, 7 are the red part nebulae spectra observed on Mar. 5, 2018, which show three various $\mathrm{H} \alpha$ emission line composited with different components. The double and multiple components may be the result of the emissions produced by the nebulae with various expansion velocities along the line of sight. 


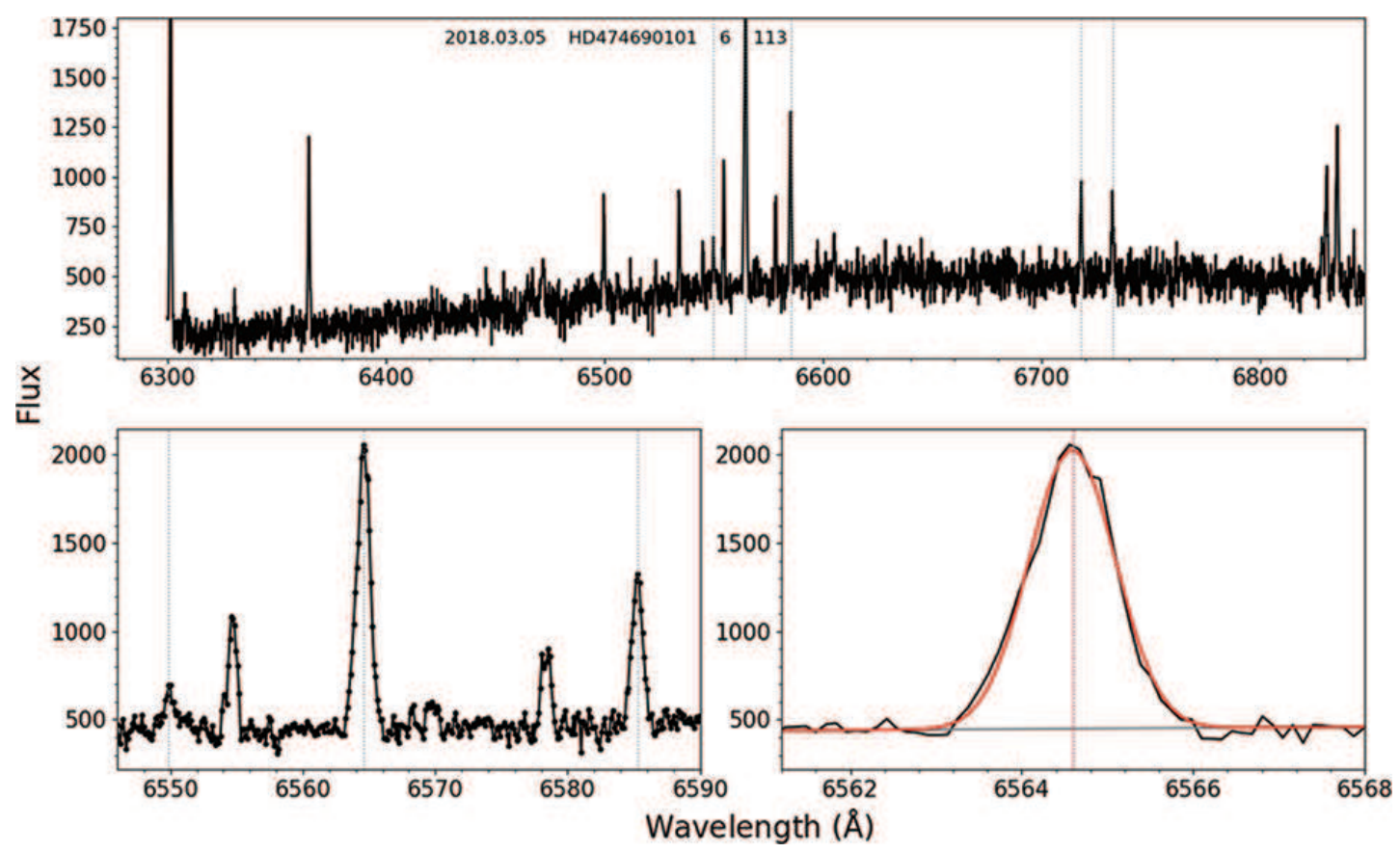

Fig. $5 \mathrm{H} \alpha$ emission line of nebulae with single-component.

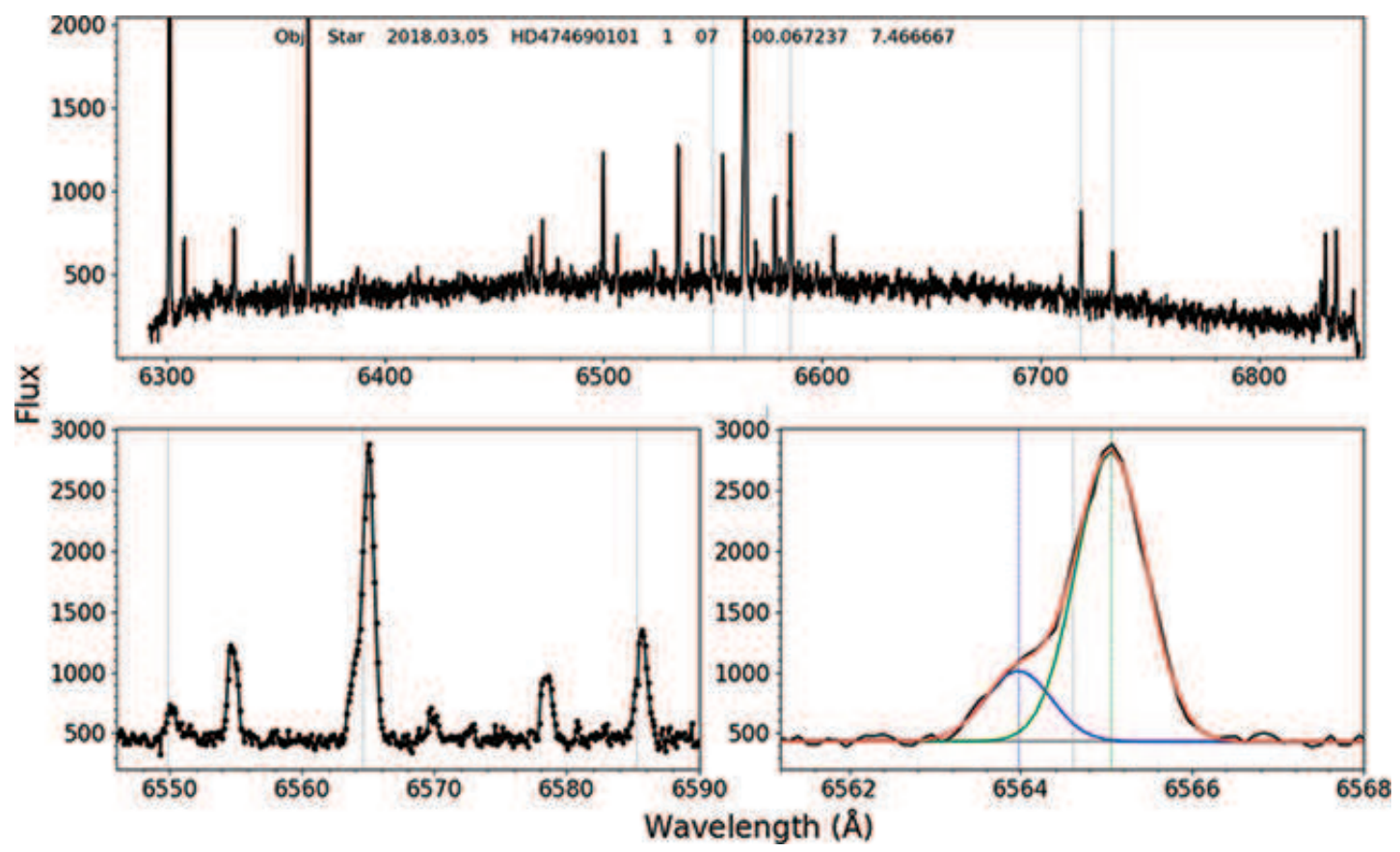

Fig. $6 \mathrm{H} \alpha$ emission line of nebulae with double-component. 


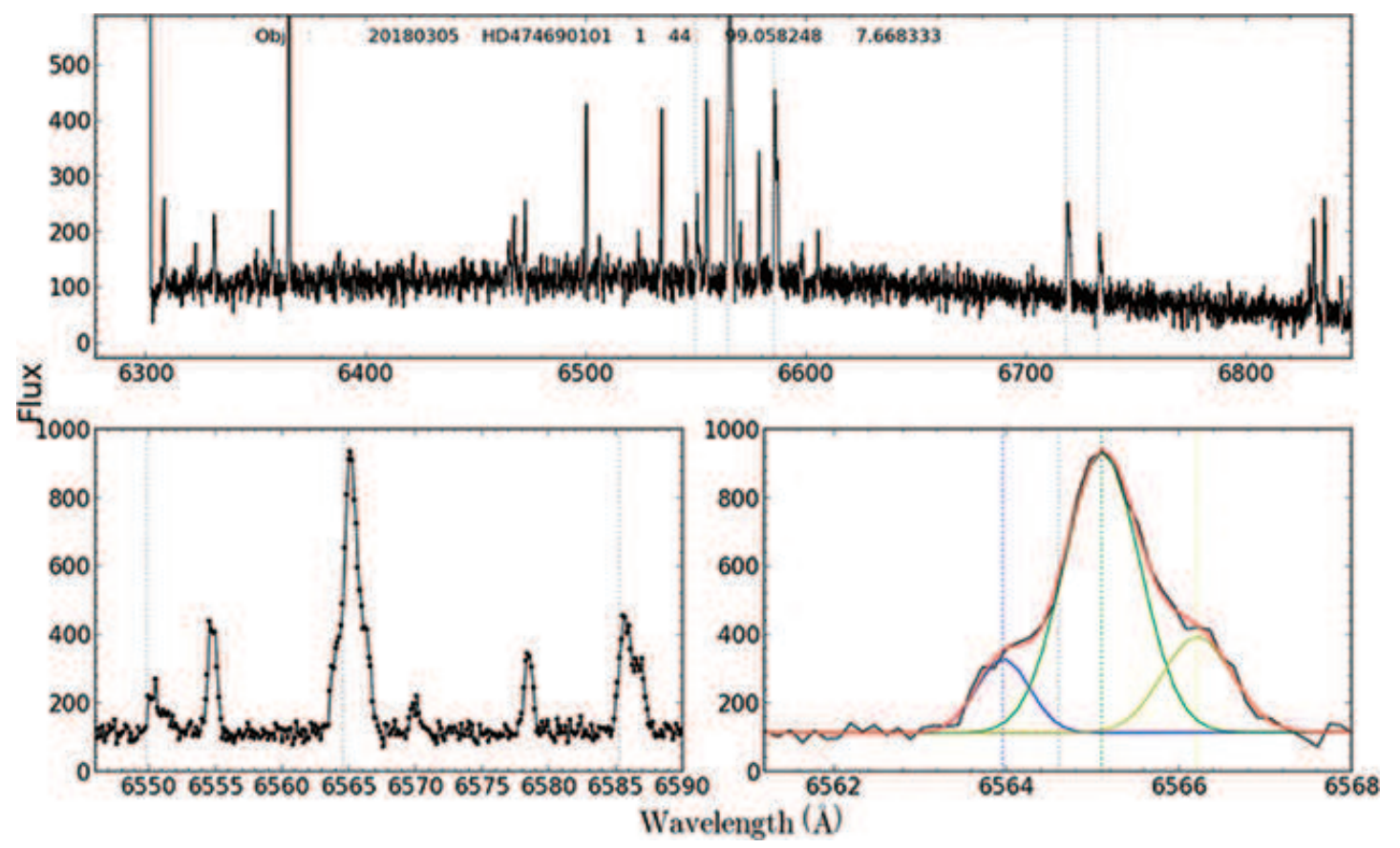

Fig. $7 \mathrm{H} \alpha$ emission line of nebulae with multi-component.

Ren et al. (2020, submit) investigated the precision of wavelength calibration and found that there are systematic deviations of radial velocities (RVs) from $\sim 0.1$ to $4 \mathrm{~km} \mathrm{~s}^{-1}$ for different plates. After by fitting 7 sky emission lines in the red part of MRS to recalibrate the wavelength, the precision of RVs is less than $1 \mathrm{~km} \mathrm{~s}^{-1}$. All the future MRS-N data will be recalibrated with this method.

\section{SUMMARY}

As the first large optical astronomical scientific device in China, LAMOST has achieved great success for the first stage of low-resolution spectroscopic survey. Since Sep. 2018, LAMOST started to conduct medium-resolution spectroscopic survey and will significantly increase the number of stellar and nebulae spectra with medium-resolution. As a sub-project of MRS, we proposed the spectral survey of Galactic nebulae with MRS. In MRS-N, the RVs, velocity dispersions, reddening and chemical compositions of most nebulae on the northern GP can be studied. Now, MRS-N has completed its two-year observation plan, which is the current largest nebulae survey over the world. In this paper, the detailed scientific goals and survey plan are presented. The first data release of MRS-N is scheduled for the third quarter of 2020 (Wu et al. 2020, in prep).

Acknowledgements This project is supported by the National Natural Science Foundation of China (Grant Nos. 11733006, 11403061, 11903048, U1631131, 11973060, U1531118, 11403037, 11225316, 11173030, 11303038, Y613991N01, U1531245 and 11833006), and the Key Laboratory of Optical Astronomy, National Astronomical Observatories, Chinese Academy of Sciences, and the Key Research Program of Frontier Sciences, CAS (Grant No. QYZDY-SSW- SLH007).

C.-H. Hsia acknowledges the supports from the Science and Technology Development Fund, Macau SAR (file Nos. 119/2017/A3, 061/2017/A2 and 0007/2019/A) and Faculty Research Grants of the Macau University of Science and Technology (No. FRG-19-004-SSI).

Guoshoujing Telescope (the Large Sky Area Multi-Object Fiber Spectroscopic Telescope LAMOST) is a National Major Scientific Project built by the Chinese Academy of Sciences. Funding 
for the project has been provided by the National Development and Reform Commission. LAMOST is operated and managed by the National Astronomical Observatories, Chinese Academy of Sciences.

\section{References}

Afflerbach, A., Churchwell, E., \& Werner, M. W. 1997, ApJ, 478, 190

Allen, G. E., Houck, J. C., \& Sturner, S. J. 2008, ApJ, 683, 773

Ambartsumian, V. A. 1954, in Liege International Astrophysical Colloquia, Vol. 5, Liege International Astrophysical Colloquia, 293

Arbutina, B. 2017, Publications de l'Observatoire Astronomique de Beograd, 97, 1

Bally, J. 2016, ARA\&A, 54, 491

Balser, D. S., Rood, R. T., Bania, T. M., \& Anderson, L. D. 2011, ApJ, 738, 27

Barentsen, G., Farnhill, H. J., Drew, J. E., et al. 2014, MNRAS, 444, 3230

Blair, W. P., \& Long, K. S. 2004, ApJS, 155, 101

Chiappini, C., Matteucci, F., \& Gratton, R. 1997, ApJ, 477, 765

Chiappini, C., Matteucci, F., \& Romano, D. 2001, ApJ, 554, 1044

Cudworth, K. M., \& Herbig, G. 1979, AJ, 84, 548

Cui, X.-Q., Zhao, Y.-H., Chu, Y.-Q., et al. 2012, Research in Astronomy and Astrophysics, 12, 1197

Davis, C. J., Gell, R., Khanzadyan, T., Smith, M. D., \& Jenness, T. 2010, A\&A, 511, A24

Deharveng, L., Peña, M., Caplan, J., \& Costero, R. 2000, MNRAS, 311, 329

Drew, J. E., Greimel, R., Irwin, M. J., et al. 2005, MNRAS, 362, 753

Dyson, J. E. 1979, A\&A, 73, 132

Esteban, C., Carigi, L., Copetti, M. V. F., et al. 2013, MNRAS, 433, 382

Esteban, C., Fang, X., García-Rojas, J., \& Toribio San Cipriano, L. 2017, MNRAS, 471, 987

Esteban, C., García-Rojas, J., Peimbert, M., et al. 2005, ApJ, 618, L95

Fernández-Martín, A., Pérez-Montero, E., Vílchez, J. M., \& Mampaso, A. 2017, A\&A, 597, A84

Fesen, R. A., Blair, W. P., \& Kirshner, R. P. 1985, ApJ, 292, 29

Fich, M., \& Silkey, M. 1991, ApJ, 366, 107

Fuentes-Masip, O., Muñoz-Tuñón, C., Castañeda, H. O., \& Tenorio-Tagle, G. 2000, AJ, 120, 752

Gerhard, O. 2002, Space Sci. Rev., 100, 129

Green, D. A. 2014, in IAU Symposium, Vol. 296, Supernova Environmental Impacts, ed. A. Ray \& R. A. McCray, 188

Hsia, C.-H., \& Zhang, Y. 2014, A\&A, 563, A63

Kennicutt, Robert C., J., \& Chu, Y.-H. 1988, AJ, 95, 720

Kohoutek, L. 2001, A\&A, 378, 843

Korotin, S. A., Andrievsky, S. M., Luck, R. E., et al. 2014, MNRAS, 444, 3301

Liu, C., Fu, J., Shi, J., et al. 2020, arXiv e-prints, arXiv:2005.07210

Long, K. 2016, What Makes Radio-detected and Optically-detected Supernova Remnants in NGC6946 Different?, HST Proposal

Luo, A. L., Zhang, H.-T., Zhao, Y.-H., et al. 2012, Research in Astronomy and Astrophysics, 12, 1243

Luo, A. L., Zhao, Y.-H., Zhao, G., et al. 2015, Research in Astronomy and Astrophysics, 15, 1095

Mao, Y.-W., Lin, L., \& Kong, X. 2018, ApJ, 853, 151

Miszalski, B., Parker, Q. A., Acker, A., et al. 2008, MNRAS, 384, 525

Monreal-Ibero, A., Relaño, M., Kehrig, C., et al. 2011, MNRAS, 413, 2242

Mundt, R. 1985, The Observatory, 105, 224

Mundt, R. 1987, Mitteilungen der Astronomischen Gesellschaft Hamburg, 70, 100

Orchiston, W., Green, D. A., \& Strom, R. 2015, New Insights From Recent Studies in Historical Astronomy: Following in the Footsteps of F. Richard Stephenson, Vol. 43

Parker, Q. A., Acker, A., Frew, D. J., et al. 2006, MNRAS, 373, 79

Pérez-Montero, E. 2017, PASP, 129, 043001

Ren, J.-J., Liu, X.-W., Chen, B.-Q., et al. 2018, Research in Astronomy and Astrophysics, 18, 111

Rudolph, A. L., Fich, M., Bell, G. R., et al. 2006, ApJS, 162, 346 
Schwartz, R. D. 1975, ApJ, 195, 631

Schwartz, R. D. 1983, Revista Mexicana de Astronomia y Astrofis, 7, 27

Searle, L. 1971, ApJ, 168, 327

Shaver, P. A., McGee, R. X., Newton, L. M., Danks, A. C., \& Pottasch, S. R. 1983, MNRAS, 204, 53

Simpson, J. P., Colgan, S. W. J., Rubin, R. H., Erickson, E. F., \& Haas, M. R. 1995, ApJ, 444, 721

Smith, M. G., \& Weedman, D. W. 1970, ApJ, 161, 33

Su, D.-Q., \& Cui, X.-Q. 2004, ChJAA (Chin. J. Astron. Astrophys.), 4, 1

Tenorio-Tagle, G., Munoz-Tunon, C., \& Cid-Fernand es, R. 1996, ApJ, 456, 264

Terlevich, R., \& Melnick, J. 1981, MNRAS, 195, 839

Vilchez, J. M., \& Esteban, C. 1996, MNRAS, 280, 720

Vučetić, M. M., Ćiprijanović, A., Pavlović, M. Z., et al. 2015, Serbian Astronomical Journal, 191, 67

Wang, L.-L., Luo, A. L., Hou, W., et al. 2018, PASP, 130, 114301

Wang, S.-G., Su, D.-Q., Chu, Y.-Q., Cui, X., \& Wang, Y.-N. 1996, Appl. Opt., 35, 5155

Woltjer, L. 1972, ARA\&A, 10, 129

Wu, C.-J., Wu, H., Hsia, C.-H., et al. 2020, Research in Astronomy and Astrophysics, 20, 033

Zhao, G., Zhao, Y.-H., Chu, Y.-Q., Jing, Y.-P., \& Deng, L.-C. 2012, Research in Astronomy and Astrophysics, 12, 723 